\title{
"We Are Talking About Practice": the Influence of Mindfulness vs. Relaxation Training on Athletes' Attention and Well-Being over High-Demand Intervals
}

\author{
Joshua D. Rooks $^{1}$ • Alexandra B. Morrison ${ }^{1} \cdot$ Merissa Goolsarran $^{1} \cdot$ Scott L. Rogers $^{2}$. \\ Amishi P. Jha ${ }^{1}$
}

Received: 2 December 2016 / Accepted: 20 March 2017 /Published online: 6 April 2017

(C) The Author(s) 2017. This article is published with open access at Springerlink.com

\begin{abstract}
We investigated the impact of short-form mindfulness training (MT) vs. relaxation training (RT) programs on sustained attention and emotional well-being in college football players $(N=100)$ during their high-demand pre-season training interval. Participants received 4 weeks of MT $(n=56)$ or RT $(n=44)$ and completed the Sustained Attention to Response Task (SART) and questionnaires assessing emotional well-being before and after the training period. Sustained attention was assessed via SART outcomes indexing performance $\left(\mathrm{A}^{\prime}\right)$, reaction time variability (intraindividual coefficient of variation (ICV)), and selfreported mind wandering and meta-awareness (Probe 1, Probe 2), while emotional well-being was assessed via the Positive and Negative Affect Schedule (PANAS), the State-Trait Anxiety Inventory (State; STAI-S), and the Center for Epidemiological Studies Depression (CES-D) scale. Overall, behavioral measures of sustained attention ( $\left.\mathrm{A}^{\prime}, \mathrm{ICV}\right)$ and self-report measures of emotional well-being (PANAS Positive, STAI-S, CES-D) declined during the training interval, suggesting that this was a highdemand interval with cognitive and emotional consequences. Further, while group effects comparing training programs were non-significant, greater engagement (i.e., practice and adherence) in MT, but not RT, predicted greater benefits, akin to protectionfrom-decline, on SART behavioral indices (A', ICV). Greater engagement in both MT and RT predicted negative change in anxiety and positive change in positive affect over the highdemand interval. These results suggest that, similar to physical training, athletes must sufficiently engage in MT and RT to experience the distinct and overlapping benefits these programs
\end{abstract}

Amishi P. Jha

a.jha@miami.edu

1 Department of Psychology, University of Miami, Miami, FL, USA

2 School of Law, University of Miami, Miami, FL, USA offer over cognitively and emotionally demanding intervals, such as pre-season athletic training.

Keywords Football $\cdot$ Sports $\cdot$ Pre-season $\cdot$ Mindfulness . Relaxation $\cdot$ Attention $\cdot$ Meditation $\cdot$ High demand

\section{Introduction}

"Concentration and mental toughness are the margins of victory."—Bill Russell

Mental toughness is regarded as a key performance asset for athletes. It is understood by those in competitive sports as the ability to sustain attention on the task-at-hand while under pressure, as well as in the face of distraction (Jones et al. 2002). Athletes are frequently offered occasions to acclimate themselves to external sources of distraction, such as practicing with simulated crowd noise and bright lights (Burton and Raedeke 2008, p. 162). However, there is a paucity of opportunity to train and strengthen their focus in the face of internal distraction. An athlete's mental toughness against internal sources of distraction, such as thoughts, emotions, and selfrelated pre-occupations, is typically viewed as part of his/her intrinsic "ability." In the current study, we propose that regardless of one's intrinsic ability, mental toughness is mutable. It may degrade over extended periods of high demand, and it may be strengthened with mental training.

Informed by cognitive neuroscience principles, we propose a definition of mental toughness herein as the capacity to sustain one's attention on the task-at-hand, in the service of protecting against distraction and its performance and emotional costs. Theoretical models emphasizing internal sources of distraction suggest that lapses in sustained attention during 
"off-task" episodes (referred to as "mind wandering") correspond with "perceptual decoupling" of attention (Smallwood and Schooler 2015), wherein attentional resources necessary for task-related cognitive and perceptual analysis are hijacked by internally generated thought. Such episodes can occur approximately $30 \%$ (Kane et al. 2007) to nearly $50 \%$ (Killingsworth and Gilbert 2010) of waking hours and can result in decreased emotional well-being (Killingsworth and Gilbert 2010). Lapses in sustained attention could have particularly deleterious effects during athletic performance, which requires keeping performance goals online while maintaining situational and interoceptive awareness (Haase et al. 2015). Thus, the margins of victory may be determined by one's success at maintaining sustained attention.

Athletes endure protracted periods of high demand, defined herein as intervals with a high frequency of physical, academic, and/or social responsibilities, as they prepare for the competition season. During high-demand intervals, the high frequency of external demands may require student athletes to expend resources of physical strength as well as cognitive and affective control to maintain optimal functioning on the field and in the classroom (Garland and Barry 1990; Smallwood et al. 2007). There is growing evidence that experiencing a prolonged interval of high demand diminishes sustained attention and increases self-reported mind wandering in college students over the course of the semester (Morrison et al. 2014), decreases emotional well-being with lower positive affect and greater negative affect over pre-deployment training in military cohorts (Jha et al. 2010), and results in performance costs on tasks of attention over a period of detention in incarcerated youth (Leonard et al. 2013). The nature of an individual's demands, performance goals, and life circumstances substantially differ between these cohorts, yet they all demonstrate attentional and/or emotional degradation associated with an extended period of high demand. Similarly, athletes must intensively prepare for competition season and, as a result, may suffer degradation during this training interval. To our knowledge, no study to date has investigated this hypothesis.

One potential approach by which to protect individuals from the attentional and emotional costs of high-demand intervals is mindfulness training (MT). Mindfulness is a mental mode characterized by attention to presentmoment experience without conceptual elaboration or emotional reactivity (Jha et al. 2010; Kabat-Zinn 1990). Mindfulness training programs provide didactic instruction and exercises to promote this mental mode. Prior studies investigating MT have demonstrated that MT can provide protective benefits to sustained attention and emotional well-being during high-demand intervals, as well as provide benefits above baseline over intervals without high demand (e.g., Jain et al. 2007; Jha et al. 2010, 2015; MacLean et al. 2010). Benefits to sustained attention, working memory, and emotional well-being have been reported in studies with long-form training (e.g., $24 \mathrm{~h}$ over 8 weeks; Jha et al. 2010, 2016). However, short-form MT programs with fewer weeks and/or less overall time investment have also been evaluated and have demonstrated benefits to performance on measures of sustained attention (Jha et al. 2015; Morrison et al. 2014).

A limitation of prior studies of short-form MT is that salutary effects are typically observed when MT groups are compared to waitlist control groups, not other active comparison programs with engaged training and homework exercises (but see Mrazek et al. 2013). This design limitation leaves open the possibility that benefits to both sustained attention and emotional well-being may not be unique to MT but may arise from effort- or expectation-related factors tied to participation in a program aimed at improving well-being. One study by Jain et al. (2007) directly tackled this possibility by testing the impact of an MT program vs. a somatic relaxation training program in students pursuing various medical degrees. Their results demonstrated that both the MT and relaxation groups had reduced psychological distress and bolstered self-reported positive affect compared to a no-treatment group. However, reductions in rumination and distraction were only found in the MT group. These results suggest that while both MT and relaxation may promote emotional well-being, MT may uniquely benefit abilities related to cognitive functioning (see Smith and Alloy 2009 for review of theoretical links between rumination and cognitive control).

The present study aimed to investigate the benefits of shortform MT vs. an active comparison group on sustained attention and emotional well-being in a cohort of college athletes. We recruited Division I college football players during their high-demand pre-season summer training interval, which takes place just prior to their participation in an intensive assessment and placement camp. All participants were randomly assigned to receive a 4-week MT program or a relaxation training (RT) program.

In addition to evaluating the comparative benefits of the MT vs. RT programs, we considered the extent to which participants engaged in the training. Training engagement was considered due to prior findings demonstrating that the protective effects of MT on sustained attention correspond to the amount of engagement in mindfulness practices (i.e., selfreported at-home practice time; Jha et al. 2016). These benefits were also demonstrated in a study of military cohorts comparing two MT variants, one emphasizing first-hand engagement in practice and another emphasizing primarily didactic content about stress and resilience (Jha et al. 2015). Results demonstrated that benefits to sustain attention were greater in the MT course emphasizing practice. Accordingly, an innovation of the present study was the examination of training engagement in the MT and RT programs as both a measure of time spent practicing as well as overall adherence - a variable taking into account individuals' time spent 
in practice, observational ratings of training behavior, and attendance in sessions.

In the present study, we investigated three main questions. (1) Do sustained attention and emotional well-being decline over the high-demand pre-season training interval experienced by participants? Given prior evidence that sustained attention and emotional well-being degrade over highdemand intervals in a variety of cohorts (e.g., Jha et al. 2015; Vasterling et al. 2006; May et al. 2015), we predicted overall decline over time. (2) If declines in sustained attention and emotional well-being are observed, are short-form MT and RT able to successfully prevent such functional decline? Based on prior studies suggesting MT-related benefits to sustained attention (Jha et al. 2016, 2015; Morrison et al. 2014; Leonard et al. 2013), as well as differences between MT and RT (Jain et al. 2007), we predicted greater stability in sustained attention in the MT group relative to the RT group, and that both MT and RT programs will bolster emotional well-being. (3) Does level of training engagement influence the effectiveness of MT and RT? We predicted that participants with greater training engagement will be better protected against declines over time.

\section{Methods}

\section{Participants}

Student athletes who were part of a Division I football program at a major university in the southeastern region of the USA participated in the present study $(N=100, M$ yo $=19.81$, $\mathrm{SD}=1.51)$ and were assigned to one of the following two training groups: MT $(n=56)$ or RT $(n=44)$. Sample size was similar to what has been reported in prior studies of mindfulness training (e.g., Jha et al. 2015) and was determined by the number of available players on the football team.

\section{Procedure}

Testing and training occurred during a 1-month period of the summer pre-season academic and football training sessions where the participants attended one to two academic courses and engaged in up to $8 \mathrm{~h}$ of weekly mandatory athletic training, which involved team-based strength and agility training, cardiovascular conditioning, and football-specific drills. The interval ended just prior to an assessment and placement camp, which included intensive physical drills and often determines players' team leadership and position roles for the season. Each student-athlete's participation was strictly voluntary, and they received no monetary compensation for their participation. The University of Miami Institutional Review Board approved the study and informed consent was obtained from each participant prior to entry into the study. All research activities were conducted in accordance with NCAA rules (2015-16 NCAA Division I Manual 2015).

Study design was single-blinded and assignment was stratified-random, with consideration of pertinent athlete-specific demographics (e.g., football position and leadership role), prior exposure to contemplative practices (e.g., yoga and meditation), and task performance at baseline. Participants were stratified along these dimensions and then randomly assigned within stratum to each group. However, group assignment was constrained by participants' coursework and team activity schedule, which led to unequal group sizes. The first data collection session (time 1 (T1)) occurred in the week prior to the onset of the training program. The second data collection session (time 2 (T2)) took place within 2 days after the last day of the training program.

\section{Mindfulness and Relaxation Training Programs}

The MT program was based on selected practices of the mindfulness-based stress reduction (MBSR) program (Kabat-Zinn 1990) and was contextualized to fit the demands and culture of the University football program. Course content and discussion surrounded mindfulness and related topics and emphasized connecting mindful awareness to footballspecific demands (e.g., distractions during performance and emotional over-reaction). Mindfulness exercises corresponding to each central topic were introduced, including mindfulness of breathing, body scan, and choiceless awareness exercises.

The RT program was based on prior stress-reduction and relaxation practices traditionally utilized in evidence-based psychotherapies (e.g., cognitive behavioral therapy; Beck 2011), and was also contextualized for the University football program. Content included information and discussion about relaxation. There was an emphasis on connecting the effects of a relaxed state (e.g., decreased muscle tension) to similar football-specific demands as the MT group. Exercises were introduced corresponding to each central topic and included pleasant place-guided imagery, progressive muscle relaxation, and listening to relaxing music.

The only overlap in topics between the training programs occurred during the final day of training. Both the MT and RT programs included a team cohesion instruction and guided practice. The team cohesion elements of the instruction were based on traditional loving-kindness meditation exercises (for review, see Shapiro and Sahgal 2012). This exercise guided participants to express a cognitive and emotional intention of kindness directed toward the self, to a fellow teammate, and to the team as a whole.

Both training programs occurred over the same interval and were offered by the same training instructor. They were matched across all delivery structure dimensions such as the number, length, and type of session. The course was referred 
to in both groups as "mindfulness and relaxation training" so that the participants were blind to their group assignment.

\section{Training Program Components}

The 9-h training programs each included $3 \mathrm{~h}$ of didactic instructor-led group training, $2.4 \mathrm{~h}$ of proctored training, and $3.6 \mathrm{~h}$ of assigned at-home individual practice.

The didactic training sessions were meetings led by the instructor once a week for $45 \mathrm{~min}$. Each didactic session included an instructor-facilitated discussion and guided training exercises that were roughly $15 \mathrm{~min}$ long. The session was focused on a single-core practice relevant to the respective training types. Participants were provided training cards to assist with topic discussion and were encouraged to keep the training cards accessible throughout the week for personal review.

Proctored training sessions were conducted on 4 days each week, either preceding or following the athletes' group physical training. Members of the research team met participants at the football training facility and provided each participant an individual MP3 player with a 12-min audio recording of a guided exercise. Guided exercises were designed and recorded by the training instructor and were uniquely tailored to each training program to complement and extend the guided exercise introduced during the week's didactic training session.

Participants were assigned at-home individual training exercises each day during the training program. These were 12min guided audio recordings provided to participants via email to download and play at their own convenience. Each week's audio recordings were identical to the guided exercises provided during the proctored training sessions for that week. Participants were instructed to record the duration of their daily at-home practice on their training cards and to submit these cards to the research team. At-home practice reports were not disclosed to the participants' coaches or the training instructor, and this was emphasized to participants to minimize social desirability biases in practice reporting.

\section{Assessment}

Testing sessions at $\mathrm{T} 1$ and $\mathrm{T} 2$ were completed in a quiet room with groups of up to 23 participants at a time. Participants sat approximately $57 \mathrm{~cm}$ from the screen of either a PC desktop or laptop computer and completed the Sustained Attention to Response Task (SART) and additional behavioral tasks beyond the scope of this report, as well as questionnaires including the following measures of emotional well-being: the Positive and Negative Affect Schedule (PANAS), the State-Trait Anxiety Inventory (State; STAI-S), and Center for Epidemiological Studies Depression (CES-D) scale described below.
SART The SART is a computer-administered go, no-go task that is typically used as a behavioral measure of sustained attention (Robertson et al. 1997). Single digits (0-9) were presented one at a time $(250 \mathrm{~ms})$, with a fixation cross following each digit $(900 \mathrm{~ms})$ that served as the inter-trial interval (Fig. 1). Participants were required to press a keyboard spacebar in response to non-target trial numbers $(0-2,4-9)$ and to withhold pressing the spacebar in response to target trial numbers (3). Responses were recorded during the stimulus display or the inter-trial interval. Target trials occurred on $5 \%$ of all trials and non-target trials on $90 \%$ of all trials, and trial order was quasi-randomized so that targets were separated by at least one other trial.

In addition, two probe questions were presented in succession and dispersed throughout the task (5\% of trials). Participants responded to the question, "Where was your attention focused just before the probe?" on a 6-point Likert scale ranging from 1 (on-task) to 6 (off-task) (Probe 1) and to the question "How aware were you of where your attention was?" on a scale from 1 (aware) to 6 (unaware) (Probe 2). After a 163-trial practice block which was not included in analysis, participants completed two experimental blocks, consisting of a total of 519 non-targets, 27 targets, and 28 sets of probes.

The primary SART performance measure of interest was indexed using A prime ( $\left.\mathrm{A}^{\prime}\right)$. $\mathrm{A}^{\prime}$ is a non-parametric measure calculated from hits (accuracy in response to targets) and false alarms (errors in response to non-targets) to measure performance while controlling for potential response biases and allowing for the difference in frequency between target and non-target trials (see Stanislaw and Todorov 1999 for A' computations). However, to provide a more complete picture of behavior on the SART, additional SART performance measures were examined, including the intraindividual coefficient of variation (ICV) and two self-report probes (Probe 1 and Probe 2). ICV was calculated by dividing the standard deviation of a participant's response time by the mean of their response for non-target trials. Greater ICV has been demonstrated to reflect greater mind wandering (Bastian and Sackur 2013). In addition, participants' responses to Probe 1 and Probe 2 were each individually averaged to index selfreported mind wandering (Probe 1) and meta-awareness (Probe 2) during the task.

PANAS The PANAS (Watson et al. 1988) is a self-report measure capturing separate dimensions of positive and negative affects. Ten items assess feelings of positive affect (e.g., enthusiastic, active, alert) and 10 assess feelings of negative affect (e.g., anger, disgust, fear). Participants rated the extent to which they felt the identified emotion in general on a 5point Likert scale from 1 (very slightly or not at all) to 5 (extremely). Positive and negative scales are calculated independently as the sum of all 10 of their respective items. The 


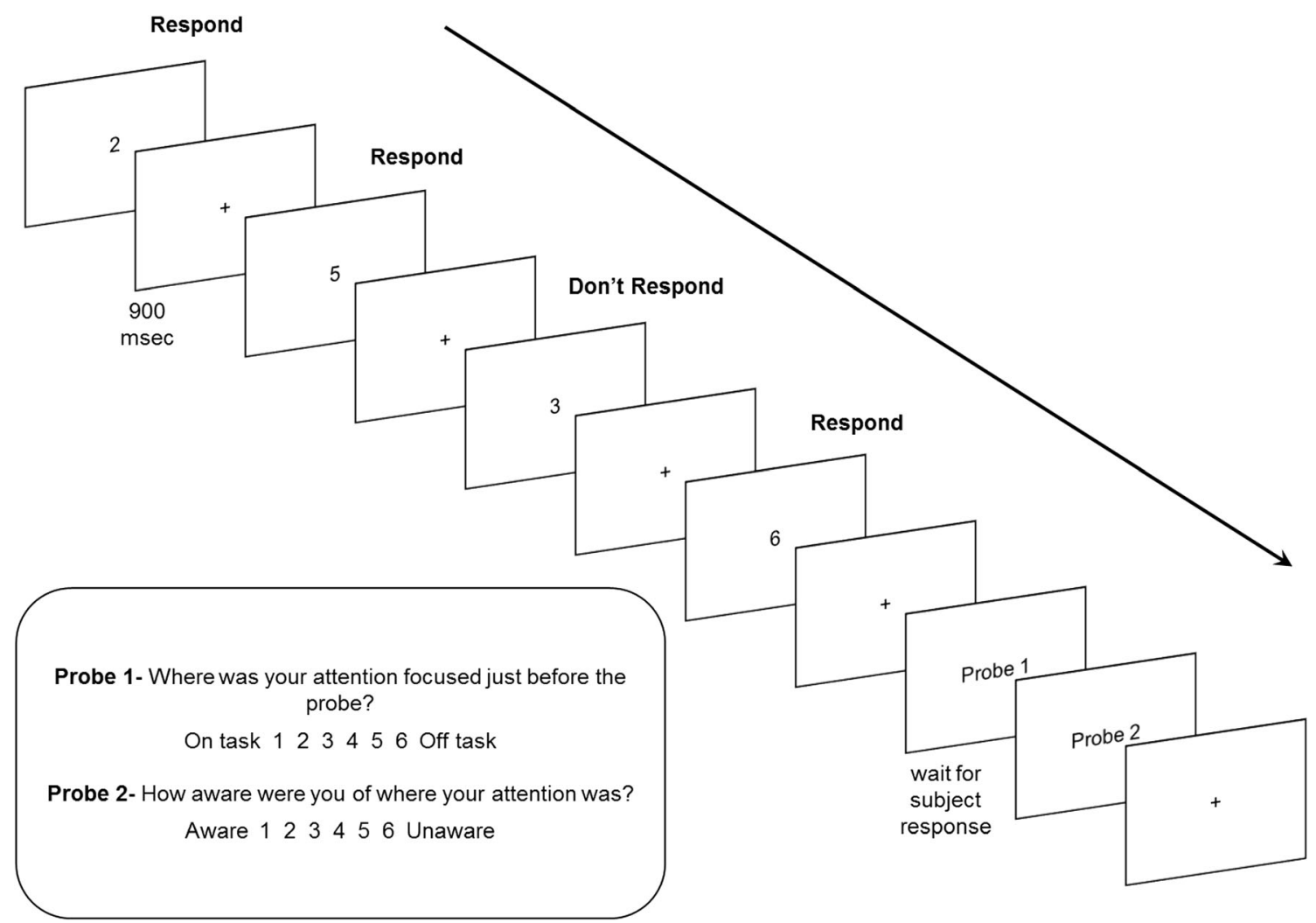

Fig. 1 Sustained Attention to Response Task (SART) schematic

two scales have demonstrated high internal consistency and stability over time as well as strong validity with measures of depression, general distress, and emotional dysfunction (Watson et al. 1988).

STAI-S The state scale of the STAI (Spielberger et al. 1970) is a 20-question self-report measure that assesses feelings of anxiety at a particular moment in time. Participants were instructed to report on a 4-point Likert scale from 1 (not at all) to 4 (very much so) the extent to which they felt levels of anxiety (e.g., tense, anxious, calm) at that moment. State scores were calculated by first reverse scoring items that reflect a state of low anxiety and then calculating the sum of all 20 items such that a greater score indicated greater levels of state anxiety. This measure has also demonstrated strong psychometric properties of reliability and validity (Spielberger et al. 1970).

CES-D The CES-D (Radloff 1977) is a 20-item self-report measure designed to assess depression in the general population. Each item required participants to rate how frequently they experienced a symptom of depression (e.g., "I felt sad" or "I felt lonely") within the past week on a 4-point Likert scale from 0 (rarely or none of the time) to 3 (most or all of the time). CES-D scores were calculated by first reverse scoring items that were worded in a positive direction, and then summing all items such that a greater score indicated greater levels of depression. This measure has demonstrated high internal consistency, adequate reliability, and strong validity with other self-report measures of depression (Radloff 1977).

\section{Training Engagement}

Practice Time Practice time was measured as the sum of participants' minutes of self-reported at-home practice and observed practice during proctored sessions throughout the 4 -week training program. At-home practice minutes were collected from participants at the beginning of the second, third, and fourth didactic training sessions. Attendance and practice completion were recorded by research team members for each participant at all proctored training sessions.

Observed Behavior Observed behavior was calculated as the average behavioral observation ratings made by members of the research team during proctored training sessions. These research team members were trained to identify six possible behaviors using a rubric to rate each participant's observable engagement in the exercise. Participants were rated using this rubric on a scale from 1 (low engagement) to 7 (high engagement). Each participant was rated by two research team members, independently, during a single proctored session at least once during the 4-week training program. Inter-rater reliability indicated fair agreement between members of the research team $(\mathrm{kappa}=0.28)$. 
Attendance Attendance was calculated as a participant's total number of didactic training sessions attended during the program. Attendance was recorded by members of the research team at the beginning of each of the training sessions.

\section{Analyses}

Statistical analyses, designed to inform our three main questions, included $t$ tests, regressions, and structural equation modeling (SEM). As a precursor to our central analyses, independent sample $t$ tests were conducted for each measure to determine whether the groups differed at $\mathrm{T} 1$.

Next, we investigated whether there was decline in measures of sustained attention and emotional well-being over the course of the high-demand pre-season training interval in all participants, regardless of group. A series of paired sample $t$ tests compared T1 to T2 for each SART measure and questionnaire score. Effect sizes (Cohen's $d_{z}$ ) were calculated according to the calculations provided by Lakens (2013).

We then evaluated the influence of training on change over this interval while taking into account the type of training (MT, RT) and an aspect of training engagement (practice time, i.e., total minutes of at-home and proctored practice). For each outcome, separately, a regression model examined whether training group, practice time, and/or the interaction of group and practice time predicted the magnitude of the change over time (T2 - T1). The training group variable was effect coded and practice time was mean centered.

We then utilized SEM to examine training engagement more comprehensively by modeling three measures of training engagement (i.e., practice time, observed behavior, and attendance) as indicators of a latent factor, "program adherence." This factor was used to address potential group differences regarding the effect of program adherence on change over time on our outcome measures. With SEM, parameter estimation was conducted with full information maximum likelihood. This estimation approach is free from the limitations of list-wise deletion and provided an intention-totreat analysis including all active participants. The practice time loading on program adherence was constrained at 1 to set the metric for the factor.

Each test model included program adherence predicting a given T2 measure score, controlled for by the respective T1 score (see Fig. 2 example model). Chi-squared difference tests were then conducted to test group differences on the structural regression paths from program adherence to each $\mathrm{T} 2$ outcome (i.e., Fig. 2, path A). Model fit comparisons were made between a model with this regression coefficient freely estimated in each group, and a nested model with this coefficient constrained to be equal. A significant chi-squared difference score suggests that effects of program adherence on outcome measures are moderated by the type of training program. All measures were assessed for normal distributions and homogeneity of variance prior to analyses. No adjustments to data were necessary. Model fit was only considered when testing for measurement invariance between training groups, as the structural aspect of the model was saturated within each group. All SEM analyses were performed with Mplus software 7th edition (Muthén and Muthén 1998-2012).

Of the 100 participants who were assigned to a training group (MT, $n=56$; RT, $n=44$ ), 15 participants (MT, $n=6$; $\mathrm{RT}, n=9)$ were lost due to attrition at T2 testing. The $t$ tests and regressions included 85 participants for PANAS, and 84 participants for CES-D and STAI, as 1 participant in RT did not complete these questionnaires at $\mathrm{T} 2$. Four participants could not be included in SART analyses. Specifically, one RT participant did not take the SART at T2. Another three participants were removed for failing to respond to any trials or for responding to only a single trial in one of the testing sessions (RT, $n=1$; MT, $n=2$ ). Therefore, 81 participants were included in $t$ tests and regressions for SART measures. SEM analyses included all 100 participants from the training program.

\section{Results}

Independent samples $t$ tests demonstrated no significant difference between MT and RT at T1 on any measures of SART performance $\left(\mathrm{A}^{\prime}, \mathrm{ICV}\right.$, Probes 1 and 2$)$ or scores on the PANAS, STAI-S, or CES-D (all $p s>0.1$ ). Self-report measures also demonstrated acceptable reliability (Cronbach's alpha $(\alpha) \geq 0.70)$ at both data collection sessions (PANAS Positive, T1 $\alpha=0.871$, T2 $\alpha=0.936$; PANAS Negative, T1 $\alpha=0.707, \mathrm{~T} 2 \alpha=0.858$; CES-D, T1 $\alpha=0.864, \mathrm{~T} 2 \alpha=0.896$; STAI-S, T1 $\alpha=0.788$, T2 $\alpha=0.764$ ).

\section{Overall Change from $\mathrm{T} 1$ to $\mathrm{T} 2$}

Table 1 includes descriptive statistics and the full results of the paired sample $t$ tests. Results showed significant decline in performance among multiple SART indices, suggesting that from $\mathrm{T} 1$ to $\mathrm{T} 2$, participants had poorer overall performance (A '; $\left.p<0.001, d_{z}=0.55\right)$ and greater variability in response times (ICV; $p<0.001, d_{z}=0.53$ ); however, self-reported mind wandering (Probe 1; $p=0.076, d_{z}=0.20$ ) and meta-awareness (Probe 2; $p=0.133, d_{z}=0.17$ ) did not change significantly.

Over this interval, participants also reported greater anxiety (STAI-S; $p=0.015, d_{z}=0.27$ ), reduced positive affect (PANAS Positive; $p<0.001, d_{z}=0.43$ ), and greater depression (CES-D; $p<0.001, d_{z}=0.53$ ). Reports specific to negative affect did not change significantly (PANAS Negative; $\left.p=0.125, d_{z}=0.17\right)$. Overall, results demonstrated a reduction in behavioral measures of sustained attention and selfreport measures of emotional well-being over the course of the high-demand training interval. 
Fig. 2 Example graphic of each SEM model of the latent factor program adherence predicting each time 2 outcome, controlling for time 1. Behave observed behavior, Attend attendance, $T 1$ time $1, T 2$ time 2

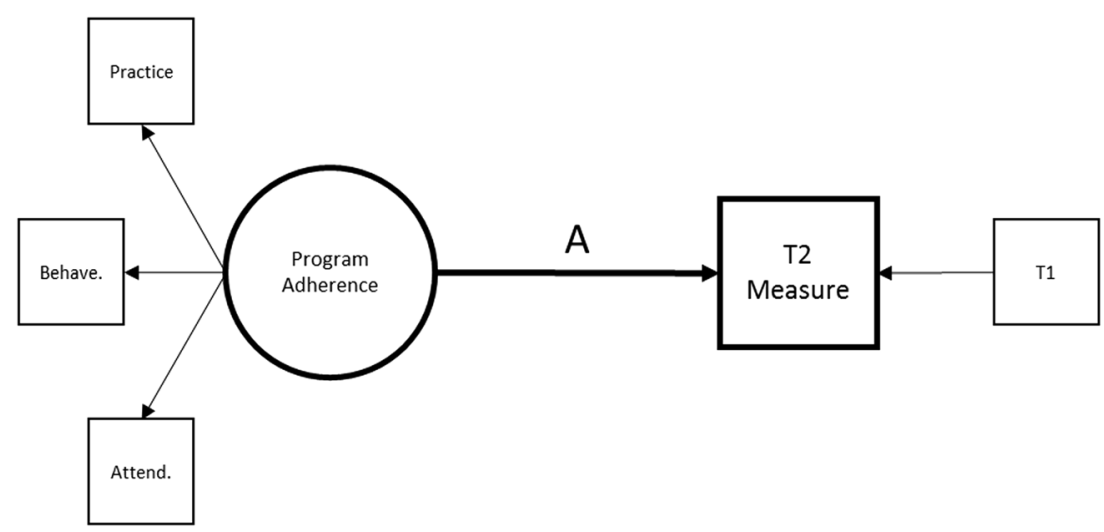

\section{Effect of Training Group and Practice Time}

Table 2 includes full results from the regression analyses for each outcome. Results indicated that the model accounted for a significant portion of the variance in change scores (T2 $\mathrm{T} 1)$ for both $\mathrm{A}^{\prime}\left(R^{2}=0.11, p=0.034\right)$ and STAI-S $\left(R^{2}=0.11\right.$, $p=0.023)$, but no other outcomes.

$\mathrm{A}^{\prime}$ did not demonstrate significant main effects of group or practice time on change from $\mathrm{T} 1$ to $\mathrm{T} 2$. However, there was a significant group by practice time interaction $(p=0.020)$, indicating that greater practice time was associated with smaller decreases or even increases in SART A' scores in the MT group (standardized coefficient $\beta=0.38, p=0.005$; see Fig. 3), while this effect was non-significant in the RT group.

STAI-S did not show a main effect of group. However, there was a main effect of practice time where greater practice time was associated with less of an increase (or even a decrease) in anxiety from $\mathrm{T} 1$ to $\mathrm{T} 2$ in both groups $(\beta=-0.30$, $p=0.015)$. Here, the interaction of group and practice time was non-significant. In sum, practice time was positively associated with change in $\mathrm{A}^{\prime}$ only in the MT group, while practice time was negatively associated with change in STAI-S among both groups.

\section{Effect of Program Adherence in MT vs. RT}

A preliminary evaluation of the latent variable demonstrated that all indicators (practice time, observed behavior, and attendance) significantly loaded onto program adherence (see Table 3). In addition, metric and scalar invariance criteria were met (Milfont and Fischer 2010), as the latent factor model fit the data when all loadings and intercepts were constrained equal between groups as indicated by a non-significant chisquared test $\left(\chi^{2}(4)=8.723, p=0.068\right)$. Further, the latent variable means were not significantly different between groups $\left(M_{\text {difference }}=-25.41, \mathrm{SE}=15.53, p=0.102\right)$.

See Table 4 for complete results of chi-squared difference tests and SEM path coefficients. Results of chi-squared difference tests demonstrated significant group differences regarding the effect of program adherence on change in $\mathrm{A}^{\prime}$ $(p=0.006)$ and ICV $(p=0.040)$ over time.

The significant differences indicated that program adherence in the MT group had a significant positive effect on change in $\mathrm{A}^{\prime}(\beta=0.51, p=0.001)$ and a significant negative effect on change in ICV $(\beta=-0.35, p=0.012)$, while both of these effects were non-significant in the RT group. To illustrate these effects, Fig. 4 shows SART performance (A' and

Table 1 Descriptive statistics separated by group and results of paired samples $t$ tests evaluating overall change from T1 to T2 in all outcomes

\begin{tabular}{|c|c|c|c|c|c|c|c|c|c|c|c|}
\hline \multirow[t]{2}{*}{ Measure } & \multicolumn{2}{|c|}{ Mindfulness $M(\mathrm{SD})$} & \multicolumn{2}{|c|}{ Relaxation $M(\mathrm{SD})$} & \multicolumn{2}{|c|}{$\begin{array}{l}\text { Mindfulness and relaxation } M \\
\text { (SD) }\end{array}$} & \multicolumn{5}{|c|}{ Paired sample $t$ tests } \\
\hline & $\mathrm{T} 1$ & $\mathrm{~T} 2$ & $\mathrm{~T} 1$ & $\mathrm{~T} 2$ & $\mathrm{~T} 1$ & $\mathrm{~T} 2$ & $t$ & $d f$ & $p$ & $d_{z}$ & $95 \% \mathrm{CI}$ \\
\hline SART A' & $0.77(0.14$ & $0.66(0.21)$ & $0.77(0.14)$ & $0.67(0.19)$ & $0.77(0.14)$ & $0.67(0.20)$ & 4.95 & 80 & $<0.001$ & 0.55 & {$[0.06,0.15]$} \\
\hline SART ICV & $0.50(0.26)$ & $0.61(0.27)$ & $0.46(0.25)$ & $0.60(0.22)$ & $0.48(0.26)$ & $0.60(0.25)$ & -4.80 & 80 & $<0.001$ & 0.53 & {$[-0.17,-0.07]$} \\
\hline SART Probe 1 & $2.02(1.08)$ & $2.26(1.31)$ & $1.76(0.96)$ & $2.20(1.33)$ & $1.91(1.04)$ & $2.23(1.31)$ & -1.80 & 80 & 0.076 & 0.20 & {$[-0.67,0.03]$} \\
\hline SART Probe 2 & $2.11(1.02)$ & $2.29(1.30)$ & $1.77(0.81)$ & $2.11(1.24)$ & $1.97(0.95)$ & $2.23(1.28)$ & -1.52 & 80 & 0.133 & 0.17 & {$[-0.58,0.08]$} \\
\hline PANAS Negative & $21.48(4.29)$ & $21.84(6.82)$ & $21.86(4.80)$ & $23.69(5.19)$ & $21.64(4.48)$ & $22.60(6.23)$ & -1.55 & 84 & 0.125 & 0.17 & {$[-2.20,0.27]$} \\
\hline PANAS Positive & $39.52(5.14)$ & $35.76(9.21)$ & $37.94(6.78)$ & $35.91(6.74)$ & $38.87(5.88)$ & $35.82(8.24)$ & 3.92 & 84 & $<0.001$ & 0.43 & {$[1.50,4.59]$} \\
\hline STAI-S & $41.14(8.55)$ & $45.43(8.15)$ & $40.86(11.25)$ & 41.91 (9.63) & $41.02(9.70)$ & $43.96(8.91)$ & -2.49 & 83 & 0.015 & 0.27 & {$[-5.28,-0.59]$} \\
\hline CES-D & $14.12(8.19)$ & $19.78(10.87)$ & $15.63(9.65)$ & $20.00(10.66)$ & $14.75(8.81)$ & $19.87(10.72)$ & -4.83 & 83 & $<0.001$ & 0.53 & {$[-7.23,-3.01]$} \\
\hline
\end{tabular}

SART Sustained Attention to Response Task, PANAS Positive and Negative Affect Schedule, STAI-S State-Trait Anxiety Inventory (State scale), CES-D Center for Epidemiological Studies Depression, ICV intraindividual coefficient of variation 
Table 2 Results from regression analyses

\begin{tabular}{|c|c|c|c|c|c|c|c|c|c|}
\hline Outcome measure $^{\mathrm{a}}$ & $B$ & $\mathrm{SE}(B)$ & $\beta$ & $t$ & $p$ & $F$ & $d f$ & $p$ & $R^{2}$ \\
\hline \multicolumn{10}{|l|}{$\mathrm{A}^{\prime}$} \\
\hline Overall model & & & & & & 3.04 & 3.77 & 0.034 & 0.11 \\
\hline Group & 0.003 & 0.022 & 0.01 & 0.13 & 0.897 & & & & \\
\hline Practice time & $<0.001$ & $<0.001$ & 0.09 & 0.75 & 0.458 & & & & \\
\hline Group $\times$ practice time & 0.001 & $<0.001$ & 0.28 & 2.37 & 0.020 & & & & \\
\hline Practice MT & 0.001 & $<0.001$ & 0.38 & 2.86 & 0.005 & & & & \\
\hline Practice RT & $<0.001$ & $<0.001$ & -0.20 & -0.97 & 0.337 & & & & \\
\hline \multicolumn{10}{|l|}{ ICV } \\
\hline Overall model & & & & & & 0.74 & 3.77 & 0.529 & 0.03 \\
\hline Group & 0.011 & 0.026 & 0.05 & 0.42 & 0.676 & & & & \\
\hline Practice time & $<0.001$ & $<0.001$ & -0.06 & -0.47 & 0.640 & & & & \\
\hline Group $\times$ practice time & $<0.001$ & $<0.001$ & 0.13 & 1.02 & 0.309 & & & & \\
\hline \multicolumn{10}{|l|}{ Probe 1} \\
\hline Overall model & & & & & & 0.23 & 3.77 & 0.877 & 0.01 \\
\hline Group & 0.126 & 0.189 & 0.08 & 0.67 & 0.507 & & & & \\
\hline Practice time & 0.001 & 0.002 & 0.07 & 0.55 & 0.585 & & & & \\
\hline Group $\times$ practice time & $<0.001$ & 0.002 & $<0.01$ & 0.01 & 0.996 & & & & \\
\hline \multicolumn{10}{|l|}{ Probe 2} \\
\hline Overall model & & & & & & 0.17 & 3.77 & 0.914 & 0.01 \\
\hline Group & 0.089 & 0.175 & 0.06 & 0.51 & 0.612 & & & & \\
\hline Practice time & 0.001 & 0.002 & 0.04 & 0.32 & 0.749 & & & & \\
\hline Group $\times$ practice time & 0.001 & 0.002 & 0.03 & 0.28 & 0.782 & & & & \\
\hline \multicolumn{10}{|l|}{ PANAS Negative } \\
\hline Overall model & & & & & & 0.78 & 3.81 & 0.508 & 0.03 \\
\hline Group & 0.66 & 0.65 & 0.11 & 1.01 & 0.315 & & & & \\
\hline Practice time & -0.003 & 0.009 & -0.05 & -0.40 & 0.691 & & & & \\
\hline Group $\times$ practice time & 0.009 & 0.009 & 0.12 & 1.00 & 0.320 & & & & \\
\hline \multicolumn{10}{|l|}{ PANAS Positive } \\
\hline Overall model & & & & & & 2.07 & 3.81 & 0.111 & 0.07 \\
\hline Group & 1.15 & 0.80 & 0.16 & 1.45 & 0.151 & & & & \\
\hline Practice time & 0.019 & 0.010 & 0.22 & 1.83 & 0.070 & & & & \\
\hline Group $\times$ practice time & 0.004 & 0.010 & 0.05 & 0.38 & 0.707 & & & & \\
\hline \multicolumn{10}{|l|}{ STAI-S } \\
\hline Overall model & & & & & & 3.37 & 3.80 & 0.023 & 0.11 \\
\hline Group & -2.23 & 1.18 & -0.21 & -1.89 & 0.062 & & & & \\
\hline Practice time & -0.038 & 0.015 & -0.30 & -2.48 & 0.015 & & & & \\
\hline Group $\times$ practice time & 0.004 & 0.015 & 0.03 & 0.25 & 0.801 & & & & \\
\hline \multicolumn{10}{|l|}{ CES-D } \\
\hline Overall model & & & & & & 0.62 & 3.80 & 0.603 & 0.02 \\
\hline Group & -0.813 & 1.11 & -0.08 & -0.73 & 0.466 & & & & \\
\hline Practice time & -0.011 & 0.014 & -0.10 & -0.79 & 0.431 & & & & \\
\hline Group $\times$ practice time & 0.008 & 0.014 & 0.06 & 0.53 & 0.597 & & & & \\
\hline
\end{tabular}

${ }^{\text {a }}$ Outcome measures represent change scores (time 2 - time 1)
ICV) for those with high practice time (practice minutes $>$ median) in both the MT $(n=24)$ and RT $(n=16)$ groups. There were no significant group differences for effects of program adherence on change in self-report SART indices (Probe 1 and Probe 2) or questionnaire scores (Positive and Negative PANAS, STAI-S, or CES-D).

When the combined group effect was examined by constraining the path to be equal between groups, the effect of program adherence was statistically significant and positive for PANAS Positive change ( $\beta=0.22, p=0.032)$ and negative for STAI-S change $(\beta=-0.42, p=0.003)$ but was not statistically significant for Probe 1 , Probe 2 , PANAS Negative, and CES-D.

Together, results from SEM analyses suggest that greater program adherence provides unique benefit to behavioral measures of sustained attention in the MT but not RT training group. However, in both groups, greater program adherence predicted benefits on select measures of emotional well-being during this high-demand interval.

\section{Discussion}

In the present study, we investigated the effects of short-form (4-week) MT vs. RT programs on sustained attention and emotional well-being in college athletes during a prolonged interval of high demand (i.e., intensive pre-season athletic training). We asked three main questions: (1) Do college athletes decline in attention and emotional well-being during this high-demand training interval? (2) If decline is observed, are short-form MT and RT able to successfully protect against such decline, and if so, are the effects of MT distinct from 


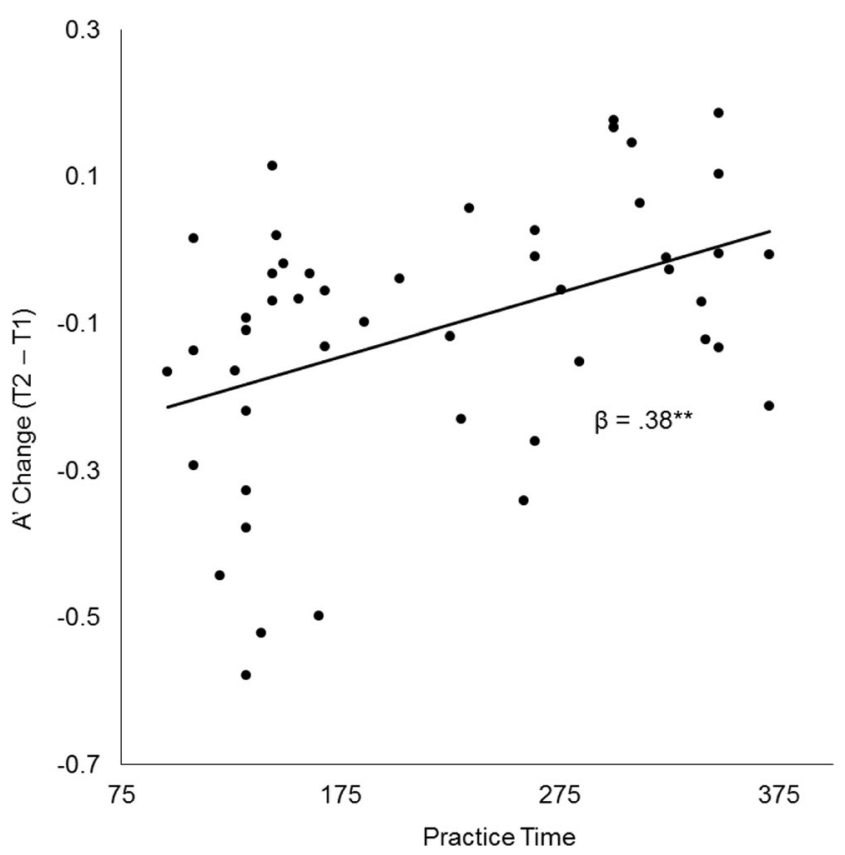

Fig. 3 Scatterplot of the association between practice time (minutes) and change (T2 - T1) in SART A' scores for mindfulness training group. T1 time $1, T 2$ time $2 ; * * p<0.01$

the effects of RT? (3) Does the pattern of training-related benefits differ as a function of participant engagement in the training programs?

In response to our first question, we found a significant trend of degradation over time in both behavioral measures of sustained attention ( $\mathrm{A}^{\prime}$ and ICV), as well as a decline in positive affect (PANAS Positive) and an increase in anxiety (STAI-S) and depression (CES-D) across all participants. While there was no significant change in self-report measures of mind wandering (Probe 1), meta-awareness (Probe 2), and negative affect (PANAS Negative), the overall pattern across multiple metrics suggests that sustained attention and emotional well-being declined over the high-demand pre-season athletic training interval. These results are consistent with previous studies demonstrating that periods of high demand can have a deleterious impact on sustained attention (Jha et al. 2015, 2016) as well as emotional well-being (Jha et al. 2010).

Regarding the second question, no significant group effects were revealed in the regression analyses of change scores, suggesting that perhaps the groups did not differ in their performance. Yet, such a conclusion would be premature without consideration of the variability in training engagement across individuals. Indeed, in both groups, training engagement varied across participants. Just as improvements in an athlete's physical performance or skill level would not be expected if he/she simply attended athletic training sessions but did not actually engage in the physical training exercises themselves, attending class meetings without fully engaging in the MT or RT exercises would not be expected to alter attention or wellbeing. By this logic, consideration of program adherence is
Table 3 Factor loadings for latent measure of program adherence

\begin{tabular}{lllll}
\hline Factor loading & \multicolumn{2}{l}{ Standardized $(\lambda)$} & Unstandardized $(\Lambda)$ & $p$ value \\
\cline { 2 - 3 } & MT & RT & & \\
\hline Practice time & 0.76 & 0.89 & 1.00 & - \\
Observed behavior & 0.64 & 0.61 & 0.008 & $<0.001$ \\
Attendance & 0.69 & 0.50 & 0.008 & $<0.001$ \\
\hline
\end{tabular}

$M T$ mindfulness training, $R T$ relaxation training

necessary to account for individual differences in adherence and to fully evaluate program effectiveness.

The first strategy taken to address the third question, regarding the impact of program adherence, involved assessing the contribution of practice time on our measures of interest. Indeed, the results from the regression analyses demonstrated that practice time predicted change in a behavioral measure of sustained attention $\left(\mathrm{A}^{\prime}\right)$ in the MT, but not RT, group. A second and more elaborated strategy taken to investigate the issue of program adherence utilized a latent factor approach that considered practice time, attendance, and second-person behavioral observation of engagement. The examination of the latent factor of program adherence found that greater training engagement predicted significant positive change in $\mathrm{A}^{\prime}$ and negative change in ICV in the MT, but not RT, group.

While the collective results from both regression and SEM suggest that MT and RT had differential benefits for SART A' and ICV scores, both programs were found to have protective effects on emotional well-being. Evidence from multiple regression models indicated a main effect of practice time, where greater practice time corresponded with less of an increase (or even a decrease) in STAI scores from T1 to T2 across all participants. Similar effects were also demonstrated in the SEM analyses where program adherence, among both groups, had a significant impact on change in STAI and PANAS Positive scores. Thus, the effects of MT and RT were comparable to each other for these measures of emotional well-being; in that, greater program engagement corresponded to greater benefits.

Our study was designed to test the effects of MT against a well-matched active comparison group (RT), an advance from previous studies of MT investigating sustained attention, in which only no-treatment control groups were included (Morrison et al. 2014; Jha et al. 2010, 2016). The present results replicate and extend findings from a study by Jha and others (Jha et al. 2016), which found similar protective effects of MT on decline in sustained attention only among those with a high degree of self-reported at-home practice time. Jha's study compared MT to a no-treatment control group and acknowledged that an alternative explanation for these protective effects may be that they are driven by applied mental effort. For instance, those who spent more time and effort 
Table 4 Results from chisquared difference tests and regression coefficients, evaluating the effect of program adherence on outcome measure change moderated by training group

\begin{tabular}{|c|c|c|c|c|c|c|c|c|}
\hline \multirow[t]{2}{*}{ Outcome measure } & \multicolumn{3}{|c|}{ Chi-squared difference test $\mathrm{t}^{\mathrm{a}}$} & \multirow[t]{2}{*}{ Group(s) } & \multicolumn{4}{|c|}{ Path $A^{b}$ regression coefficients } \\
\hline & $\Delta \chi^{2}$ & $d f$ & $p$ & & $B$ & SE & $p$ & $\beta$ \\
\hline \multirow[t]{2}{*}{$\mathrm{A}^{\prime}$} & 7.68 & 1 & 0.006 & MT & 0.001 & $<0.001$ & 0.001 & 0.51 \\
\hline & & & & RT & $<0.001$ & $<0.001$ & 0.254 & -0.19 \\
\hline \multirow[t]{2}{*}{ ICV } & 4.19 & 1 & 0.040 & MT & -0.001 & 0.001 & 0.012 & -0.35 \\
\hline & & & & RT & 0.001 & 0.001 & 0.393 & 0.16 \\
\hline Probe 1 & 0.53 & 1 & 0.466 & MT and RT & 0.001 & 0.003 & 0.792 & 0.04 \\
\hline Probe 2 & 0.39 & 1 & 0.534 & MT and RT & 0.001 & 0.003 & 0.771 & 0.04 \\
\hline PANAS Negative & 0.14 & 1 & 0.706 & MT and RT & -0.002 & 0.012 & 0.886 & -0.02 \\
\hline PANAS Positive & 0.26 & 1 & 0.609 & MT and RT & 0.031 & 0.014 & 0.032 & 0.22 \\
\hline STAI-S & 0.25 & 1 & 0.614 & MT and RT & -0.050 & 0.017 & 0.003 & -0.42 \\
\hline CES-D & 0.77 & 1 & 0.380 & MT and RT & -0.043 & 0.022 & 0.053 & -0.24 \\
\hline
\end{tabular}

${ }^{\text {a }}$ Chi-squared difference test values represent the difference in model fit between models with the structural regression paths from program adherence to each $\mathrm{T} 2$ outcome free to vary vs. constrained equal between training groups

${ }^{\mathrm{b}}$ See Fig. 2 for the graphic of path A in SEM model engaging in at-home MT practices may have also applied greater mental effort at $\mathrm{T} 2$ testing. The concerns regarding mental effort are less applicable in the present study due to the inclusion of an active comparison group (i.e., RT). If the effects of applied mental effort, and motivation effects more broadly, were the sole factors driving the protective effects of training, we would expect to see similar patterns demonstrated by both the MT and RT groups, when program engagement was considered. Yet, we do not see similar SART performance effects for MT and RT; instead, only MT was found to have benefits commensurate with practice engagement.

The present study also contributes to a growing literature regarding mindfulness- and relaxation-based interventions. Our results are in line with previous work showing that benefits to emotional well-being were seen following both MT and RT while benefits to cognitive functioning were unique to MT (Jain et al. 2007). While prior work examined cognitive functioning via a questionnaire measuring ruminative and distracting thoughts (Jain et al. 2007), the present study shows benefits to an objective measure of sustained attention. Further support for differentiation between the effects of MT and RT was found in a study by Creswell et al. (2016), which examined a high-stress population of unemployed adults. When compared to RT, MT was shown to increase the resting-state functional connectivity between regions implicated in executive control and mind wandering. At a 4-month post-training follow-up, there was also evidence of reduced biomarkers of inflammatory disease in the MT group compared to the RT group. As such, an increasing number of studies suggest unique benefits of MT beyond what is found in a wellmatched control group that does not emphasize mindfulnessrelated content.

One additional advantage in this study was that training engagement was measured by a latent factor of program adherence. The advantages of this approach compared to a single self-report measure of practice time include decreased

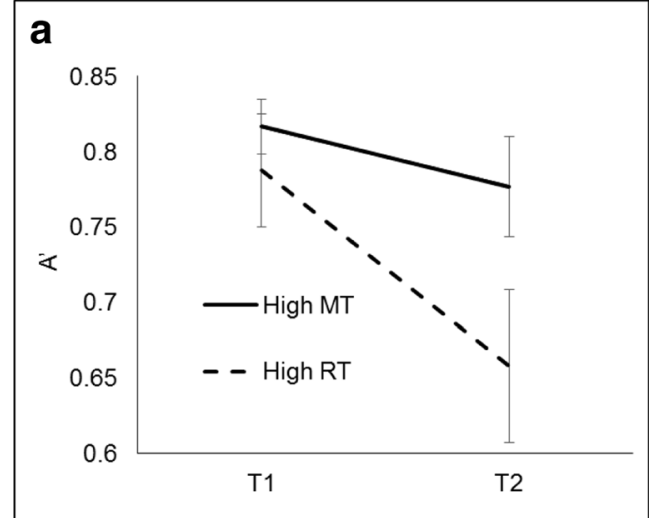

Fig. 4 Line graph representations of SART performance for $\mathbf{a} \mathrm{A}^{\prime}$ and $\mathbf{b}$ ICV at T1 and T2 for participants with high practice time in both the MT and RT groups. High MT mindfulness training group participants with

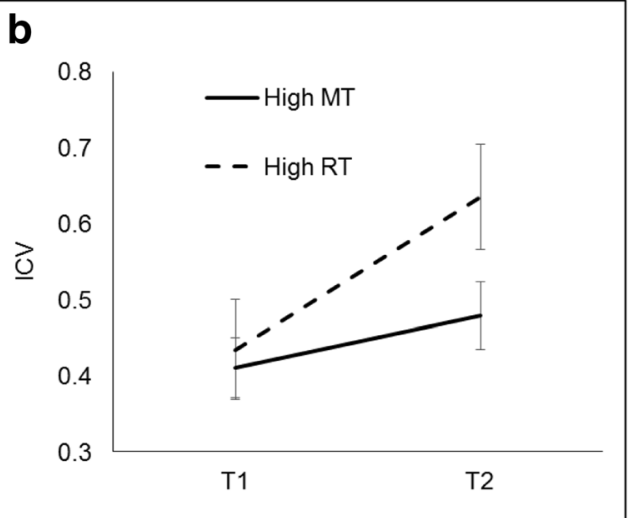

practice time $>$ median, high $R T$ relaxation training group participants with practice time $>$ median, $T 1$ time $1, T 2$ time 2 
vulnerability to demand effects and biases in self-report data, greater comprehensiveness in the measurement of this construct (e.g., first-, second-, and third-person reports; see Davidson and Kaszniak 2015), reduced measurement error, and greater statistical power (Kline 2011). To the best of our knowledge, this is the first study to measure training engagement in MT with a latent factor. However, future research is warranted to further test and replicate these models, as group sample size was relatively small for factor analyses (MacCallum et al. 2001) and tests for measurement invariance (Schmitt and Kuljanin 2008).

Given that prior studies of the SART have demonstrated that, in the absence of a high-demand interval, the task is stable over repeated testing (Jha et al. 2015), we argue that task instability cannot account for our results. Participants showed poorer overall performance $\left(\mathrm{A}^{\prime}\right)$ and increases in reaction time variability (ICV), which indicate poorer attention at the end of the 4-week interval than at the beginning. We interpret these patterns over the month-long training interval as related to the depleting effects of the attentional demands required during preparation for the athletic season. According to a resource depletion framework of attention, repeated exposure to high-demand tasks depletes cognitive resources over time and result in poorer performance on subsequent tasks with overlapping demands (Persson et al. 2007). Athletes in the present study had a rigorous schedule including daily strength and speed training, multiple weekly footballspecific meetings, end-of-semester final exams, and fitness testing. As such, in line with the resource depletion framework, these demands may have depleted cognitive resources over time.

We posit that MT may protect against such attentional resource depletion by bolstering individuals' attention over the 4-week program. Concentrative MT exercises such as those included in the MT program herein aim to repeatedly engage aspects of executive control necessary to sustain attention to the task at hand while limiting lapses in attention. The result of high engagement in the MT program may have been a bolstering of executive control processes, which consequently protected behavior on a measure of attention (the SART) from decline over an interval including mental, physical, and affective demands.

While the current study overcomes several pitfalls of past work in this topic area, there are multiple limitations that warrant future research. One limitation is that we were not able to include a randomized no-treatment or waitlist control group. Such a group would have provided a baseline assessment of sustained attention and emotional well-being over this highdemand training interval. However, as noted above, previous studies have reported similar findings of the deleterious effects of prolonged high-demand intervals on sustained attention and emotional well-being in groups not receiving training (e.g., Jha et al. 2010, 2016). Future studies are necessary to determine whether the decline observed in the present study would be comparable to what would be observed in a wellmatched, no-treatment cohort. Another limitation of the present study is that the research team involved in behavioral observations of participants' proctored training sessions may have been vulnerable to bias in their ratings due to the singleblind nature of the study. Future studies are encouraged to utilize raters with no prior contact with participants and no knowledge of the training group identities. An additional limitation is that our sample size is small relative to typical analyses utilizing SEM, where studies often include 200 or more participants (Kline 2011). A consequence of a smaller sample in SEM is that it may lead to instability in parameter estimates with full information maximum likelihood estimation.

No significant group effects in $\mathrm{T} 1-\mathrm{T} 2$ change scores on measures of sustained attention and well-being were found in our initial analyses, which did not take program engagement into consideration. This is a pattern consistent with other studies finding null effects from MT interventions (McMillan et al. 2002; Morone et al. 2009). Reporting null findings is important as it has been suggested that there is a bias toward the publication of positive results over null results (CoronadoMontoya et al. 2016). Despite the lack of group effects in the initial analyses, the interactions found between group and program engagement suggest to us that the null effect of group is not indicative of a lack of benefits to MT. Rather, our findings highlight the importance of considering individual differences in program engagement when assessing the influence of MT on sustained attention and emotional well-being.

While the present study did not explicitly investigate brain injury, the athletic cohort of football players participating in this study is from a population with vulnerability to brain injury (Selassie et al. 2013). The measure of sustained attention used in this study (i.e., the SART) was first developed as a neurobehavioral phenotype of frontal lobe and white matter damage following traumatic brain injury (Robertson et al. 1997). As such, this study provides some evidence that a neurobehavioral phenotype of brain injury may benefit from MT. Furthermore, the results of the present study are broadly consistent with other findings demonstrating the benefits of MT on attention regulation and mental fatigue following traumatic brain injury (Azulay et al. 2013; Johansson et al. 2015). In light of these findings, future research should include largescale studies to offer MT to populations vulnerable to traumatic brain injury or concussive injury and examine its putative brain-protective effects in those who may, unfortunately, end up with such brain injury.

In sum, we have demonstrated that sustained attention and emotional well-being are at risk during periods of high demand, such as a period of intensive athletic training. Greater engagement in MT was associated with protection from decline in sustained attention during this training period. And, greater engagement in MT and RT protected against decline in 
well-being. Although NBA All-Star and Naismith Memorial Hall of Famer, Allen Iverson, may disagree, the present study suggests that when considering the potential moderators of training benefits, it is very important that we "talk about practice." Thus, just as physical exercise must be performed with regularity to train the body for performance success, mental exercises must be practiced with regularity to benefit the athlete's attention and well-being.

Acknowledgements We thank the University of Miami Football Program for their service and participation in this project. We thank Joseph Dunne, Sam Warn, and Matt Gosselin for their assistance with data collection. We thank Dr. Maria Llabre for her statistical expertise. This project was funded by the Department of Army Grant \#WX81XWH-11-2-0044 to APJ.

Open Access This article is distributed under the terms of the Creative Commons Attribution 4.0 International License (http:// creativecommons.org/licenses/by/4.0/), which permits unrestricted use, distribution, and reproduction in any medium, provided you give appropriate credit to the original author(s) and the source, provide a link to the Creative Commons license, and indicate if changes were made.

\section{References}

2015-16 NCAA Division I Manual. (2015). Indianapolis: The National Collegiate Athletic Association

Azulay, J., Smart, C. M., Mott, T., \& Cicerone, K. D. (2013). A pilot study examining the effect of mindfulness-based stress reduction on symptoms of chronic mild traumatic brain injury/postconcussive syndrome. The Journal of Head Trauma Rehabilitation, 28(4), 323-331. doi:10.1097/HTR.0b013e318250ebda.

Bastian, M., \& Sackur, J. (2013). Mind wandering at the fingertips: automatic parsing of subjective states based on response time variability. Frontiers in Psychology, 4. doi:10.3389/fpsyg.2013.00573.

Beck, J. S. (2011). Cognitive behavior therapy: basics and beyond (2nd ed.). New York: Guilford Press.

Burton, D., \& Raedeke, T. D. (2008). Sport psychology for coaches. Champaign: Human Kinetics.

Coronado-Montoya, S., Levis, A. W., Kwakkenbos, L., Steele, R. J., Turner, E. H., \& Thombs, B. D. (2016). Reporting of positive results in randomized controlled trials of mindfulness-based mental health interventions. PloS One, 11(4). doi:10.1371/journal.pone.0153220

Creswell, J. D., Taren, A. A., Lindsay, E. K., Greco, C. M., Gianaros, P. J., Fairgrieve, A., et al. (2016). Alterations in resting state functional connectivity link mindfulness meditation with reduced interleukin6: a randomized controlled trial. Biological Psychiatry. doi:10.1016/ j.biopsych.2016.01.008 (Online first).

Davidson, R. J., \& Kaszniak, A. W. (2015). Conceptual and methodological issues in research on mindfulness and meditation. American Psychologist, 70(7), 581-592. doi:10.1037/a0039512.

Garland, D. J., \& Barry, J. R. (1990). Sport expertise: the cognitive advantage. Perceptual and Motor Skills, 70(3, Pt 2), 1299-1314. doi: 10.2466/PMS.70.4.1299-1314.

Haase, L., May, A. C., Falahpour, M., Isakovic, S., Simmons, A. N., Hickman, S. D., et al. (2015). A pilot study investigating changes in neural processing after mindfulness training in elite athletes. Frontiers in Behavioral Neuroscience, 9, 229. doi:10.3389/fnbeh. 2015.00229.

Jain, S., Shapiro, S. L., Swanick, S., Roesch, S. C., Mills, P. J., Bell, I., \& Schwartz, G. R. (2007). A randomized controlled trial of mindfulness meditation versus relaxation training: effects on distress, positive states of mind, rumination, and distraction. Annals of Behavioral Medicine, 33(1), 11-21. doi:10.1207/ s15324796abm3301_2.

Jha, A. P., Stanley, E. A., Kiyonaga, A., Wong, L., \& Gelfand, L. (2010). Examining the protective effects of mindfulness training on working memory and affective experience. Emotion, 10(1), 54-64. doi:10. 1037/a0018438.

Jha, A. P., Morrison, A. B., Dainer-Best, J., Parker, S., Rostrup, N., \& Stanley, E. (2015). Minds "at attention": mindfulness training curbs attentional lapses in military cohorts. PloS One, 10(2), 1-19. doi:10. 1371/journal.pone.0116889.

Jha, A. P., Morrison, A. B., Parker, S. C., \& Stanley, E. A. (2016). Practice is protective: mindfulness training promotes cognitive resilience in high-stress cohorts. Mindfulness. doi:10.1007/s12671-015-0465-9.

Johansson, B., Bjuhr, H., Karlsson, M., Karlsson, J., \& Rönnbäck, L. (2015). Mindfulness-based stress reduction (MBSR) delivered live on the internet to individuals suffering from mental fatigue after an acquired brain injury. Mindfulness, 6(6), 1356-1365. doi:10.1007/ s12671-015-0406-7.

Jones, G., Hanton, S., \& Connaughton, D. (2002). What is this thing called mental toughness? An investigation of elite sport performers. Journal of Applied Sport Psychology, 14, 205-218.

Kabat-Zinn, J. (1990). Full catastrophe living: using the wisdom of your body and mind to face stress, pain and illness. New York: Delacorte.

Kane, M. J., Brown, L. H., McVay, J. C., Silvia, P. J., Myin-Germeys, I., \& Kwapil, T. R. (2007). For whom the mind wanders, and when: an experience-sampling study of working memory and executive control in daily life. Psychological Science, 18(7), 614-621. doi:10. 1111/j.1467-9280.2007.01948.x.

Killingsworth, M. A., \& Gilbert, D. T. (2010). A wandering mind is an unhappy mind. Science, 330, 932. doi:10.1126/science.1192439.

Kline, R. B. (2011). Principles and practice of structural equation modeling. New York: Guilford Press.

Lakens, D. (2013). Calculating and reporting effect sizes to facilitate cumulative science: a practical primer for $t$-tests and ANOVAs. Frontiers in Psychology, 4, 863. doi:10.3389/fpsyg.2013.00863.

Leonard, N. R., Jha, A. P., Casarjian, B., Goolsaran, M., Garcia, C., Cleland, C. M., Gwadz, M. V., \& Massey, Z. (2013). Mindfulness training improves attentional task performance in incarcerated youth: a group randomized controlled intervention trial. Frontiers in Psychology, 4(792), 1-12. doi:10.3389/fpsyg.2013.00792.

MacCallum, R. C., Widaman, K. F., Preacher, K. J., \& Hong, S. (2001). Sample size in factor analysis: the role of model error. Multivariate Behavioral Research, 36(4), 611-637. doi:10.1207/ S15327906MBR3604 06.

MacLean, K. A., Ferrer, E., Aichele, S. R., Bridwell, D. A., Zanesco, A. P., Jacobs, T. L., et al. (2010). Intensive meditation training improves perceptual discrimination and sustained attention. Psychological Science, 21(6), 829-839. doi:10.1177/0956797610371339.

May, R. W., Bauer, K. N., \& Fincham, F. D. (2015). School burnout: diminished academic and cognitive performance. Learning and Individual Differences, 42, 126-131. doi:10.1016/j.lindif.2015.07.015.

McMillan, T., Robertson, I. H., Brock, D., \& Chorlton, L. (2002). Brief mindfulness training for attentional problems after traumatic brain injury: a randomised control treatment trial. Neuropsychological Rehabilitation, 12(2), 117-125. doi:10.1080/09602010143000202.

Milfont, T. L., \& Fischer, R. (2010). Testing measurement invariance across groups: applications in cross-cultural research. International Journal of Psychological Research, 3(1), 111-121.

Morone, N. E., Rollman, B. L., Moore, C. G., Li, Q., \& Weiner, D. K. (2009). A mind-body program for older adults with chronic low back pain: results of a pilot study. Pain Medicine, 10(8), 13951407. doi:10.1111/j.1526-4637.2009.00746.x.

Morrison, A. B., Goolsarran, M., Rogers, S. L., \& Jha, A. P. (2014). Taming a wandering attention: short-form mindfulness training in 
student cohorts. Frontiers in Human Neuroscience, 7(897), 1-12. doi:10.3389/fnhum.2013.00897.

Mrazek, M. D., Franklin, M. S., Phillips, D. T., Baird, B., \& Schooler, J. W. (2013). Mindfulness training improves working memory capacity and GRE performance while reducing mind wandering. Psychological Science, 24(5), 776-781. doi:10.1177/ 0956797612459659.

Muthén, L. K., \& Muthén, B. O. (1998-2012). Mplus user's guide (Seventh ed.). Los Angeles: Muthén \& Muthén.

Persson, J., Welsh, K. M., Jonides, J., \& Reuter-Lorenz, P. A. (2007). Cognitive fatigue of executive processes: interaction between interference resolution tasks. Neuropsychologia, 45(7), 1571-1579. doi: 10.1016/j.neuropsychologia.2006.12.007.

Radloff, L. S. (1977). The CES-D scale: a self-report depression scale for research in the general population. Applied Psychological Measurement, 1(3), 385-401. doi:10.1177/014662167700100306.

Robertson, I. H., Manly, T., Andrade, J., Baddeley, B. T., \& Yiend, J. (1997). "Oops!": performance correlates of everyday attentional failures in traumatic brain injured and normal subjects. Neuropsychologia, 35(6), 747-758. doi:10.1016/S0028-3932(97) 00015-8.

Schmitt, N., \& Kuljanin, G. (2008). Measurement invariance: review of practice and implications. Human Resource Management Review, 18(4), 210-222. doi:10.1016/j.hrmr.2008.03.003.

Selassie, A. W., Wilson, D. A., Pickelsimer, E. E., Voronca, D. C., Williams, N. R., \& Edwards, J. C. (2013). Incidence of sportrelated traumatic brain injury and risk factors of severity: a population-based epidemiologic study. Annals of Epidemiology, 23(12), 750-756. doi:10.1016/j.annepidem.2013.07.022.
Shapiro, S. L., \& Sahgal, M. (2012). Loving-kindness. In T. G. Plante (Ed.), Religion, spirituality, and positive psychology: understanding the psychological fruits of faith (pp. 109-126). Santa Barbara: Praeger/ABC-CLIO.

Smallwood, J., \& Schooler, J. W. (2015). The science of mind wandering: empirically navigating the stream of consciousness. Annual Review of Psychology, 66, 487-518. doi:10.1146/annurev-psych-010814015331.

Smallwood, J., Fishman, D. J., \& Schooler, J. W. (2007). Counting the cost of an absent mind: Mind wandering as an underrecognized influence on educational performance. Psychonomic Bulletin \& Review, 14(2), 230-236. doi:10.3758/BF03194057.

Smith, J. M., \& Alloy, L. B. (2009). A roadmap to rumination: a review of the definition, assessment, and conceptualization of this multifaceted construct. Clinical Psychology Review, 29(2), 116-128. doi:10. 1016/j.cpr.2008.10.003.

Spielberger, C. D., Gorsuch, R. L., \& Lushene, R. E. (1970). STA1 manual for the State-Trait Anxiety Inventory. Palo Alto: Consulting Psychologist Press.

Stanislaw, H., \& Todorov, N. (1999). Calculation of signal detection theory measures. Behavior Research Methods, Instruments \& Computers, 31(1), 137-149. doi:10.3758/BF03207704.

Vasterling, J. J., Proctor, S. P., Amoroso, P., Kane, R., Heeren, T., \& White, R. F. (2006). Neuropscyhological outcomes of army personnel following deployment to the Iraq war. JAMA, 296(5), 519-529. doi:10.1001/jama.296.5.519.

Watson, D., Clark, L. A., \& Tellegen, A. (1988). Development and validation of brief measures of positive and negative affect: The PANAS scales. Journal of Personality and Social Psychology, 54(6), 1063-1070. doi:10.1037/0022-3514.54.6.1063. 